\title{
EFECTOS TOXICOLOGICOS DE CUATRO PLANTAS SOBRE EL GORGOJO DEL MAIZ SITOPHILUS ZEAMAIS MOTSCHULSKY 1855 (COLEOPTERA: CURCULIONIDAE) Y SOBRE EL GORGOJO DE LAS GALLETAS STEGOBIUM PANICEUM(LINNAEUS 1761)(COLEOPTERA: ANOBIIDAE) EN PERU
}

\author{
TOXICOLOGICAL EFFECTS OF FOUR PLANTS ON CORN BORER \\ SITOPHILUS ZEAMAIS MOTSCHULSKY 1855 (COLEOPTERA: \\ CURCULIONIDAE) AND ON DRUGSTORE BEETLE STEGOBIUM PANICEUM \\ (LINNAEUS 1761) (COLEOPTERA: ANOBIIDAE) IN PERU
}

\author{
José Iannacone*1, Hildebrando Ayala*2 \& Amid Román*3 \\ * Laboratorio de Ecofisiología Animal. Facultad de Ciencias Naturales y Matemática. \\ Universidad Nacional Federico Villarreal. Calle San Marcos 383, Pueblo Libre, Lima, Perú. \\ 1Email: joseiannacone@yahoo.es \\ ${ }^{2}$ Email: hildebrandoayala@hotmail.com \\ 33Email: amid_el_v@yahoo.com
}

\begin{abstract}
RESUMEN
Se evaluó el efecto biocida de cuatro plantas: culantro Coriandrum sativum L. (Apiaceae), tara Caesalpinia spinosa (Mol.) Kuntze (Fabaceae), amor seco Bidens pilosa L. (Asteraceae) y saúco Sambucus peruviana HBK (Caprifoliaceae) sobre adultos de Sitophilus zeamais Motschulsky 1885 (Curculionidae) y Stegobium paniceum (Linnaeus 1761) (Anobiidae) en bioensayos de mortandad bajo condiciones de laboratorio. A las máximas concentraciones empleadas $(20 \% \mathrm{p} / \mathrm{v}), 1 \mathrm{~s}$ extractos acuosos de C. sativum, C. spinosa, B. pilosa y S. peruviana no mostraron efectos significativos en comparación con el control sobre ambas especies de gorgojos. Sólo los polvos secos de C. sativum produjeron un $25 \%$ de mortandad en $S$. zeamais, y en cambio, $C$. sativum ocasionó sobre $S$. paniceum, un $15 \%$ de mortandad, a las más altas dosis ensayadas $\left(1,6 \mathrm{~g} \cdot 10 \mathrm{~g}\right.$ de maíz $\left.^{-1}\right)$. En adición, al evaluar C. spinosa, bajo infusión acuosa $(20 \% \mathrm{p} / \mathrm{v})$ sobre $S$. zeamais se produjo un 17,5\% de mortandad. Sin embargo, ninguna de las cuatro plantas bajo todas las formulaciones evaluadas obtuvo más de $40 \%$ de mortandad en las dos especies de gorgojos en comparación con el control. Se analizan las posibilidades de integración de las plantas biocidas en el control de gorgojos plagas de productos agrícolas almacenados.
\end{abstract}

Palabras claves: Bidens pilosa, Caesalpinia spinosa, Coriandrum sativum, extractos vegetales, gorgojos, insecticidas botánicos, Sambucus peruviana.

\section{ABSTRACT}

The biocide effect of four plants: coriander (Coriandrum sativum L. [Apiaceae]), sping holdback (Caesalpinia spinosa (Mol.) Kuntze [Fabaceae]), hairy beggarticks (Bidens pilosa L. [Asteraceae]) and elderberry (Sambucus peruviana HBK [Caprifoliaceae]), on adults of Sitophilus zeamais Motschulsky 1855 (Curculionidae) and Stegobium paniceum (Linnaeus 1761) (Anobiidae) in mortality bioassays at laboratory conditions were evaluated. At the highest concentrations of watery extracts $(20 \% \mathrm{w} / \mathrm{v})$ used to $C$. sativum, C. spinosa, B. pilosa and S. peruviana did not show significant effects in comparison to control on both species of weevils. Only dry dusts of $C$. sativum showed $25 \%$ of mortality of $S$. zeamais and however $C$. spinosa produced $15 \%$ of mortality of $S$. paniceum at the higher concentrations assayed $\left(1.6 \mathrm{~g} \cdot 10 \mathrm{~g} \mathrm{of} \mathrm{corn}{ }^{-1}\right)$. In addition, when C. spinosa, was evaluated in infusion watery $(20 \% \mathrm{w} / \mathrm{v})$ on $S$. zeamais, $17.5 \%$ of mortality was found. However, none of the plants in all formulations employed had more than $40 \%$ mortality on two species of weevils in comparison to control. The possibilities of integration of biocide plants in control of agricultural stored product pests are analyzed.

Keywords: Bidens pilosa, Caesalpinia spinosa, Coriandrum sativum, vegetable extracts, weevils, botanical insecticides, Sambucus peruviana. 
Efecto biosida de cuatro plantas sobre S. zeamais y S. paniceum: IANNACONE, J. ET AL.

\section{INTRODUCCION}

El rápido crecimiento poblacional de la humanidad ha acarreado la necesidad de la aparición de nuevas técnicas con el propósito de aumentar la producción de alimentos, así como también minimizar las pérdidas existentes, sin perjudicar la calidad de los mismos. En este sentido los cereales tienen gran relevancia, constituyéndose como una principal fuente de alimentos, y destacándose entre ellos el maíz Zea mays L. (Clavijo \& Pérez 2000; Tavares 2002).

Entre el 30 y $40 \%$ de la producción de maíz en América Latina, se pierde durante el almacenamiento (Lagunes 1994). Una de las causas son las plagas de los granos almacenados, entre ellos, el gorgojo del maíz Sitophilus zeamais Motschulsky 1855 y el gorgojo de las galletas, del pan o de las drogas Stegobium paniceum (Linnaeus 1761), los cuales atacan a granos agrícolas en el campo y durante el almacenamiento (Lagunes et al. 1985; Morgan et al. 1998; Platt et al. 1998; Dahno et al. 2001; Trematerra \& Sciaterra 2004). Para minimizar estas pérdidas, normalmente se utilizan insecticidas químicos sintéticos, pero con frecuencia conducen a problemas de resistencia en los insectos, contaminación del ambiente y presencia de residuos en alimentos. Además, con cierta frecuencia el uso de insecticidas convencionales en áreas rurales implica un riesgo elevado debido al desconocimiento sobre su uso adecuado (Silva et al. 2003; Obeng-Ofori \& Amiteye 2005). En el mundo y en el Perú, el control químico con insecticidas sintéticos es el más empleado para el control de gorgojos plagas de almacenes (White \& Leesch 1996; Rajendran \& Parveen 2005), por ser efectivo, de bajo costo y de fácil manejo (Harein \& Davis 1992). Sin embargo, los insecticidas químicos generan efectos negativos en los seres humanos por su alta capacidad de bioacumulación y su poder residual prolongado. Una alternativa a este problema es el uso de productos naturales derivados de plantas, generalmente biodegradables y no producen un desequilibrio en el ecosistema (Iannacone \& Reyes 2001; Iannacone \& Lamas 2003a).

El uso de plantas con propiedades insecticidas es una técnica ancestral usada en Africa y América Central por cientos de años, pero con la aparición de los insecticidas sintéticos su empleo ha sido descontinuado (Bisset 2002; Iannacone \& Lamas 2003a), pero en los últimos años está teniendo nuevamente mayor importancia (Lagunes et al. 1985).

Coriandrum sativum L. (Apiaceae) es una planta herbácea, con propiedades antimicrobianas contra bacterias gram-positivas, gram-negativas y Saccharomyces cerevisiae Myen ex E.C. Hansen 1883 a partir de sus extractos crudos y de sus fracciones (Delaquis et al. 2002; Singh et al. 2002; Kubo et al. 2004). Se ha registrado efectos antialimentarios sobre Deroceras reticulatum (Müller 1774) (Limacidae: Pulmonada) y efectos biocidas sobre larvas de Aedes fluviatilis (Lutz 1904) (Diptera: Culicidae) (Consoli et al. 1988; Birkett et al. 2004).

Caesalpinia spinosa (Mol.) Kuntze (Fabaceae) es una planta arbórea nativa del Perú, con usos medicinales, tintórea, tánica (cortezas curtientes), y apta para el manejo de rebrotes y que tolera la aridez (Brack 2003).

Bidens pilosa L. (Asteraceae) es una planta anual, de 30 a $100 \mathrm{~cm}$ de altura, ampliamente distribuida en las regiones tropicales y subtropicales del mundo (Lastra \& Ponce de León 2001; Pysek 2004). En los estudios fitoquímicos de B. pilosa se han aislado alcaloides, saponinas, charconas, glucósidos fenilpropanoides, poliacetilenos, diterpenos y flavonoides (Abajo et al. 2004).

Sambucus peruviana HBK (Caprifoliaceae) es una planta usada como frutal o alimenticia para el hombre, es productora de madera de alta calidad, es apta para sombra, es medicinal y apta para manejo de rebrotes (Hernández et al. 1999; Brack 2003). En el Perú, durante un programa de prospección de plantas promisorias, se ha evaluado el efecto biocida de floripondio Brugmansia candida Pers. (Solanaceae), higuerilla Ricinus communis L. (Euphorbiaceae), guanábana Annona muricata L. (Annonaceae), palta Persea americana Miller (Lauraceae), quinua Chenopodium quinoa Willdenow (Chenopodiaceae) y Lantana camara L. (Verbenaceae) sobre adultos de $S$. zeamais y $S$. paniceum, encontrándose a $P$. americana, C. quinoa y L. camara con mayor efectividad para el control de estos dos insectos (Iannacone \& Quispe 2004; Iannacone et al. 2004).

El objetivo de este trabajo fue evaluar, bajo condiciones de laboratorio, el efecto insecticida de polvos y extractos de C. sativum (Apiaceae), $C$. spinosa (Fabaceae), B. pilosa (Asteraceae) y $S$. peruviana (Caprifoliaceae) sobre adultos de $S$. zeamais y de $S$. paniceum, para su posterior empleo en el manejo integrado de estas dos especies de insectos. 


\section{MATERIALES Y METODOS}

Todos los bioensayos se realizaron en el Laboratorio de Ecofisiología Animal de la Facultad de Ciencias Naturales y Matemática de la Universidad Nacional Federico Villarreal (LEA-FCCNM-UNFV) entre agosto del 2004 a febrero del 2005.

\section{INSECTOS}

Las crianzas de $S$. zeamais y de $S$. paniceum fueron iniciadas a partir de adultos procedentes del Mercado Jorge Chávez, La Victoria, Lima, Perú, y alimentadas con maíz variedad amarillo duro para pollo y variedad cancha. Posteriormente los individuos fueron trasladados al laboratorio, separados por especie y colocados en envases plásticos de 1 $\mathrm{L}$ de capacidad, y mantenidos a una temperatura aproximada de $25^{\circ} \mathrm{C} \pm 3{ }^{\circ} \mathrm{C}$, para favorecer la reproducción, oviposición y obtención de los adultos requeridos para realizar los bioensayos toxicológicos (Throne 1994).

\section{Plantas}

Las plantas utilizadas en los ensayos toxicológicos provienen de distintas localidades del Perú: $C$. sativum (Apiaceae), C. spinosa (Fabaceae), B. pilosa (Asteraceae) y S. peruviana (Caprifoliaceae) (Tabla I). C. sativum proviene del valle del Rímac, Carapongo, San Juan de Lurigancho, Chosica, Lima, Perú; en cambio C. spinosa y S. peruviana fueron colectadas de un parque del distrito de Pueblo Libre, Lima, Perú. Finalmente, B. pilosa fue obtenida de un mercado procedente de la Provincia de Huacho, Lima, Perú.

$150 \mathrm{~g}$ de hojas de cada especie fueron secadas en estufa a $37^{\circ} \mathrm{C}$, por $70 \mathrm{~h}$ aproximadamente, hasta obtener un peso constante por la pérdida de agua. Posteriormente las hojas fueron trituradas en un mortero (Haldenwanger $\left.{ }^{\circledR}\right)$ y el residuo obtenido fue pasado secuencialmente por 2 tamices de $500 \mu$ y $250 \mu$. Las muestras fueron envasadas en frascos de vidrio color ámbar para evitar la fotolisis, rotuladas, y guardadas a temperatura de $25^{\circ} \mathrm{C} \pm 3^{\circ} \mathrm{C}$ hasta el día a ser utilizadas en los bioensayos (Iannacone \& Lamas 2003b).

TABLA I. Especies de plantas evaluadas sobre el gorgojo del maíz Sitophilus zeamais y sobre el gorgojo de la galleta Stegobium paniceum.

TABLE I. Plant species evaluated on corn borer Sitophilus zeamais and on drugstore beetle Stegobium paniceum.

\begin{tabular}{lccc}
\hline Nombre científico & Nombre común & Familia & Parte utilizada \\
\hline Coriandrum sativum & culantro & Apiaceae & Hojas \\
Caesalpinia spinosa & tara & Fabaceae & Hojas \\
Bidens pilosa & amor seco & Asteraceae & Hojas \\
Sambucus peruviana & saúco & Caprifoliaceae & Hojas \\
\hline
\end{tabular}

\section{BioenSAYOS}

EN SECO: Se colocaron 10 individuos de cada especie de gorgojo en envases cuadrangulares de plástico con cuatro réplicas. La distribución del polvo seco de las plantas se realizó de la siguiente manera: 0,1 g, 0,2 g, 0,4 g, 0,8 g y 1,6 g por cada 10 g de maíz en cada una de las 4 replicas, y cinco dosis más un control (Iannacone et al. 2004); los insectos fueron controlados cada $24 \mathrm{~h}$ durante 5 días $(=120 \mathrm{~h})$, según la técnica sugerida por Mazzonetto \& Vendramim (2003).

EN ACUOSO: El extracto botánico acuoso crudo fue preparado con agua destilada $(\mathrm{pH} \quad 6 \pm 0,5)$ a cinco concentraciones, y filtrados a través de un papel fino (Whatman ${ }^{\circledR} \mathrm{N}^{\circ} 5$, USA). Sólo se usaron extractos acuosos que habían sido recientemente preparados (no más de $48 \mathrm{~h}$ ), debido a que microorganismos fúngicos pudieran afectar la calidad de los mismos (Iannacone \& Lamas 2003b). Se colocaron $10 \mathrm{~g}$ de maíz previamente remojados por 5 seg por cada concentración y cada tratamiento, y se agregaron 10 individuos de cada especie de gorgojo en cada envase con las concentraciones de 1,25\%, 2,5\%, $5 \%, 10 \%$ y $20 \%$, respectivamente, con cuatro réplicas más un control, evaluados cada $24 \mathrm{~h}$ durante 5 días (= $120 \mathrm{~h}$ ), para obtener el efecto de mortalidad. Para los extractos de cocción e infusión en agua destilada se prepararon a las mismas concentraciones que los extractos acuosos a temperatura ambiente, siguiendo las recomendaciones de Iannacone et al. (2004). 
Efecto biosida de cuatro plantas sobre S. zeamais y S. paniceum: IANNACONE, J. ET AL.

DiSEÑO EXPERIMENTAL Y TRATAMIENTO ESTADÍSTICO Las pruebas de toxicidad aguda se evaluaron en concentraciones nominales para cada planta a través de un ANDEVA, con el modelo aditivo lineal, empleando un diseño en bloque completamente aleatorizado (DBCR) de 6 concentraciones-dosis $\mathrm{x} 4$ repeticiones. Los datos fueron transformados a arcoseno (porcentaje de mortalidad de adultos/ 100) ${ }^{0,5}$ antes del análisis, para estabilizar el error de la varianza. En el caso de existir diferencias significativas entre las repeticiones y entre los tratamientos se hicieron pruebas de diferencias verdaderamente significativas (DVS) de Tukey. Solo cuando en los bioensayos se encontraron mortalidades diferentes de cero en el control los análisis estadísticos se realizaron con los valores ajustados según la fórmula de Abbott (Iannacone \& Lamas 2003b). Se empleó el paquete estadísti- co SPSS en español, versión 12,0 para el cálculo de la estadística descriptiva e inferencial.

\section{RESULTADOS}

A las máximas concentraciones empleadas (20\%), los extractos acuosos de C. sativum, C. spinosa, B. pilosa y $S$. peruviana no mostraron efectos significativos para ambas especies de gorgojos (Tablas II y III). Sólo los polvos secos de C. spinosa produjeron un $25 \%$ de mortandad en S. zeamais, y C. sativum ocasionó sobre $S$. paniceum un $15 \%$ de mortandad, a las más altas concentraciones ensayadas $\left(1,6 \mathrm{~g} \cdot 10 \mathrm{~g}\right.$ de maíz $\left.{ }^{-1}\right)$ (Tablas II y III). También, al evaluar C. spinosa bajo infusión acuosa sobre $S$. zeamais se encontró un $17,5 \%$ de mortandad (Tabla II).

TABLA II. Porcentaje de mortalidad de la más alta concentración de las plantas evaluadas sobre el gorgojo del maíz Sitophilus zeamais a $120 \mathrm{~h}$ de exposición.

TABLE II. Percentage of mortality at the highest concentration of the plants evaluated on corn borer Sitophilus zeamais at $120 \mathrm{~h}$ exposure.

\begin{tabular}{lllll}
\hline Tratamiento & \multicolumn{4}{c}{ mortalidad $(\%)$} \\
\cline { 2 - 5 } & $\mathrm{A}$ & $\mathrm{B}$ & $\mathrm{C}$ & $\mathrm{D}$ \\
\hline Caesalpinia spinosa & 25 & 0 & 17,5 & 0 \\
Bidens pilosa & 0 & 0 & 0 & 0 \\
Sambucus peruviana & 0 & 0 & 0 & 0 \\
Control & 0 & 0 & 0 & 0 \\
\hline
\end{tabular}

A: Polvo seco $\left(1,6 \mathrm{~g} \cdot 10 \mathrm{~g}\right.$ de maíz $\left.{ }^{-1}\right)$.

B: Extracto acuoso $(20 \%)$.

C: Extracto infusión $(20 \%)$

D: Extracto cocción $(20 \%)$.

TABLA III. Porcentaje de mortalidad de la más alta concentración de las plantas evaluadas sobre el gorgojo de la galleta Stegobium paniceum a $120 \mathrm{~h}$ de exposición.

TABLE III. Percentage of mortality at the highest concentration of the plants evaluated on drugstore beetle Stegobium paniceum at $120 \mathrm{~h}$ exposure.

\begin{tabular}{lllll}
\hline Tratamiento & \multicolumn{3}{l}{ mortalidad (\%) } & \\
\cline { 2 - 5 } & $\mathrm{A}$ & $\mathrm{B}$ & $\mathrm{C}$ & $\mathrm{D}$ \\
\hline Coriandrum sativum & 15 & 0 & 0 & 0 \\
Bidens pilosa & 0 & 0 & 0 & 0 \\
Control & 0 & 0 & 0 & 0 \\
\hline A
\end{tabular}

A: Polvo seco $\left(1,6 \mathrm{~g} \cdot 10 \mathrm{~g}\right.$ de maíz $\left.^{-1}\right)$.

B: Extracto acuoso $(20 \%)$.

C: Extracto infusión $(20 \%)$.

D: Extracto cocción (20\%). 


\section{DISCUSIONY YONCLUSIONES}

La literatura científica señala diversas plantas promisorias con propiedades biocidas sobre gorgojos de granos almacenados (Oliveira et al. 2003). Así, una concentración de 0,05\% de semillas de plantas tropicales de Basella alba $\mathrm{L}$. (Basellaceae), Operculina turpethum L. (Convolvulaceae) y Calotropis gigantea R.Br. (Asclepiadaceae) retrasaron el desarrollo y redujeron la emergencia de adultos de S. zeamais en $62 \%, 95$ $\%$ y $70 \%$, respectivamente. También se observó actividad biológica de estas plantas a una concentración menor de 0,01\% (Haque et al. 2000). Bajo condiciones de laboratorio, los polvos secos de hojas de Chenopodium ambrosioides L. evaluados sobre S. zeamais y Sitophilus granarius (Linnaeus 1758), utilizando granos de maíz mezclados con polvo de hojas de C. ambrosioides a concentraciones de 0,05-0,80\%, obtuvieron una mortalidad de $100 \%$ con la dosis de $6,4 \%$ después de $48 \mathrm{~h}$ de exposición (Tapondjou et al. 2002).

El polvo seco procedente de los frutos de $C$. ambro-sioides es altamente tóxico vía inhalación, actuando por contacto e ingesta en adultos, y los granos tratados con el polvo de este fruto afectan su fase inmadura, pero los extractos acuosos de hojas, flores y frutos no mostraron ningún efecto tóxico. Estos resultados concuerdan con el hecho que en $C$. spinosa se observa mayor mortalidad de $S$. zeamais con los polvos secos que con el extracto de infusión (Tabla II). En especies congenéricas de Caesalpinia se han reportado compuestos mayor-mente liposolubles con propiedades biocidas como bilobetinas, diterpenos, limonoides y casalpinos (Kuria et al. 2001; Woldemichael et al. 2003), lo cual explicaría por qué no se encontraron efectos tóxicos en los extractos acuosos (en frío y decocción) de $C$. spinosa sobre $S$. zeamais, pero sí en los polvos secos de $C$. spinosa (que presentan en conjunto los compuestos liposolubles e hidrosolubles) (Tabla II). Sin embargo, el ina-decuado conocimiento de la composición fotoquímica específica de C. spinosa no nos permite explicar por qué en infusión acuosa sobre $S$. zeamais produjo un 17,5\% de mortandad (Tabla II). En los limonoides de diversas plantas, entre ellas las del género Caesalpinia, también se han reportado efectos insecticidas (Obeng-Ofori \& Amiteye 2005). En C. sativum se han reportado compuestos con propiedades biocidas como el burneol, linalool, cineole, cimene, terpineol y terpinolene (Kubo et al. 2004). Posiblemente en conjunto, el mismo efecto de los polvos secos de C. spinosa podría ayudar a explicar el efecto toxicológico de los polvos secos de C. sativum sobre S. paniceum ( $\mathrm{Su}$ 1986) (Tabla III). Los efectos tóxicos de los compuestos terpenoides pueden ser atribuidos a un mecanismo de inhibición competitiva reversible por la acetilcolinesterasa, al ocupar el sitio hidrofóbico del centro activo de la enzima (Obeng-Ofori \& Amiteye 2005).

Es conocido que el procedimiento de extracción puede influenciar la cantidad de los componentes activos en el extracto final. Dependiendo del origen de la planta, los constituyentes químicos pueden variar entre plantas individuales debido a factores genéticos y ambientales. También el estado de desarrollo de la planta en la cosecha, el proceso de secado y las técnicas de almacenamiento pueden afectar la concentración de los ingredientes activos (Hördegen et al. 2003; Singh et al. 2003). Final-mente, ninguna de las plantas en las formulaciones evaluadas desde la perspectiva como insecticida (Tablas II-III), fueron consideradas altamente promisorios, ya que no produjeron un $40 \%$ de mortandad a $120 \mathrm{~h}$ de exposición a la máxima concentración evaluada, criterio considerado por Lagunas (1994) para la selección de plantas con propiedades biocidas sobre gorgojos de granos almacenados.

\section{AGRADECIMIENTOS}

Los autores expresan su agradecimiento al Laboratorio de Ecofisiología Animal de la Facultad de Ciencias Naturales y Matemática de la Universidad Nacional Federico Villarreal por su apoyo a la presente investigación. A Javier Alvarez, Julissa Ordóñez, Omar Leyva, Famela Vásquez, Alexander Corzo por su apoyo en el manejo de los gorgojos y en la preparación de las plantas evaluadas. El presente trabajo fue presentado en las Jornadas Internacionales Rioplatenses de Toxicología y Ecotoxicología, 1-2 de junio, 2005, Montevideo, Uruguay. 
Efecto biosida de cuatro plantas sobre S. zeamais y S. paniceum: IANNACONE, J. ET AL.

\section{BIBLIOGRAFIA}

Abajo, C., M.A. Boffill, J. Del Campo, M.A. Méndez, Y. GonzÁlez, M. Mitjans \& M.P. Vinardell. 2004. In vitro study of the antioxidant and immunomodulatory activity of aqueous infusion of Bidens pilosa. J. Ethnopharmacol. 93: 319-323.

Birkett, M.A., C.J. Dodds, I.F. Henderson, L.D. Leake, J.A. Pickett, M.J. Selby \& P.J. Watson. 2004. Antifeedant compounds from three species of Apiaceae active against the field slug, Deroceras reticulatum (Müller). J. Chem. Ecol. 30: 563-576.

Bisset, N. 2002. War and hunting poisons of the New World. Part 1. Notes on the early history of curare. J. Ethnopharmacol. 86: 1-26.

Brack, A.E. 2003. Perú: Diez mil años de domesticación. Ed. Bruño, Lima, Perú. 160 p.

Clavijo, S. \& G. Pérez. 2000. El Maíz en Venezuela. pp. 345-361. Cap. 6. In Protección y Sanidad Vegetal. Sec 2. Insectos Plagas del Maíz. Ed. Fundación Polar.

Consoli, R.A., N.M. Mendes, J.P. Pereira, B. De S. Santos \& M.A. Lamounier. 1988. Effect of several extracts derived from plants on the survival of larvae of Aedes fluviatilis (Lutz) (Diptera: Culicidae) in the laboratory. Mem. Inst. Oswaldo Cruz 83: 87-93.

Danho, M., C. Gaspar \& E. Haubru. 2001. The impact of grain quantity on the biology of Sitophilus zeamais Motschulsky (Coleoptera: Curculionidae): oviposition, distribution of eggs, adult emergence, body weight and sex ratio. J. Stored Prod. Res. 38: 259-266.

Delaquis, P.J., K. Stanich, B. Girard \& G. Mazza. 2002. Antimicrobial activity of individual and mixed fraction of dill, cilantro, coriander and eucalyptus essentials oils. Int. J. Food Microbiol. 74: 101109.

Haque, M., H. Nakakita, H. Ikenaga \& N. Sota. 2000. Development inhibiting activity of some tropical plants against Sitophilus zeamais. J. Stored Prod. Res. 36: 281-287.

Harein, P \& R. Davis. 1992. Control of stored-grain insects. pp. 491-534. In D.B. Sauer (Ed.). Storage of cereal grains and their products. Ed. St. Paul: American Association of Cereal Chemists.

Hernández, N.E., M.L. TereschuK, S.M. Grancelli, M.V. Quarenghi \& R.L. Abdala. 1999. Biological activity of flavonoids from Sambucus peruviana (Caprifoliaceae). Biocell 23: 45.

IANNACONE, J. \& M. REYES. 2001. Efecto de la rotenona y neem sobre Bemisia tabaci Gennadius (Homoptera: Aleyrodidae) y Liriomyza huidobrensis Blanchard (Diptera: Agromyzidae) plagas del tomate en el Perú. Agronomía Trop. 51: 65-79.

IAnNacone, J. \& G. Lamas. 2003a. Plantas biocidas usadas en el control de la polilla de la papa, Phthorimaea operculella (Zeller) (Lepidoptera: Gelechiidae). Rev. per. Ent. 43: 79-87.
Iannacone, J. \& G. Lamas. 2003b. Efectos toxicológicos de extractos de molle (Schinus molle) y lantana (Lantana camara) sobre Chrysoperla externa (Neuroptera: Chrysopidae), Trichogramma pintoi (Hymenoptera: Trichogrammatidae) y Copidosoma koehleri (Hymenoptera: Encyrtidae), en el Perú. Agri. Tec. 63: 347-360.

Iannacone, J., H. Ayala, J. Alvarez, O. Leyva \& E. BAJAlque, E. 2004. Cuatro plantas biocidas sobre Sitophilus zeamais y Stegobium paniceum en el Perú Wiñay Yachay (Perú) 8:16-27.

IAnNacone, J. \& C. Quispe. 2004. Efecto insecticida de dos extractos vegetales sobre el gorgojo del maíz, Sitophilus zeamais Motschulsky, 1855 (Coleoptera: Curculionidae) en Perú. Rev. per. Ent. 44: 81-87.

Kubo, I., K. Fujita, A. Kubo, K. Nihei \& T. Ogura. 2004. Antibacterial activity of coriander volatile compounds against Salmonella cholerasuis. J. Agric. Food Chem. 52: 3329-3332.

Kuria, K.A.M., S. De Coster, G. Muriuki, W. Masengo, I. Kibwage, J. Hoogmartens \& G.M. Laekeman. 2001. Antimalarial activity of Ajuga remota Benth (Labiatae) and Caesalpinia volkensii Harms (Caesalpiniaceae): in vitro confirmation of ethnopharmacological use. J. Ethnopharmacol. 74: 141-148.

LAGUNES, T .A. 1994. Extractos, polvos vegetales y polvos minerales para el combate de plagas del maíz y del fríjol en la agricultura de subsistencia. Memorias del Colegio de Postgraduados USAIDCONACYT-BORUCONSA. Montecillo. Texcoco. México. 32 pp.

Lagunes, T. A., R. Domínguez \& J.C. Rodríguez. 1985. Plagas del Maíz en la Mesa Central de México. Colegio de Postgraduados. Universidad Autónoma Chapingo. Documento de Trabajo. Montecillo. Texcoco. México. 100 pp.

Lastra, H.A. \& L.R. Ponce De León. 2001. Bidens pilosa Linné. Rev. Cubana Plant. Med. 1: 28-33.

Mazzonetto, F. \& J.D. Vendramim. 2003. Efeito de pós de origem vegetal sobre Acanthoscelides obtectus (Say) (Coleoptera: Bruchidae) em feijão armazenado. Neotropical Entomol. 32: 145-149.

Morgan, C., J. Sherington, I. Gudrups \& N.S. Bowden. 1998. The assessment of potential attractants to beetle pests: improvements to laboratory pitfall bioassay methods. J. Stored Prod. Res. 34: 59-74.

Obeng-Ofori, D. \& S. Аmiteye. 2005. Efficacy of mixing vegetable oils with pirimiphos-methyl against the maize weevil, Sitophilus zeamais. Motschulsky in stored maize. J. Stored Prod. Res. 41: 57-66.

Oliveira, S., J.D. Vendramim, J.I. Ribeiro \& J.B. Dos Santos. 2003. Bioatividade de diversos pós de origem vegetal em relação a Sitophilus zeamais Mots. (Coleoptera: Curculionidae). Ciên Agr. 27: 1231-1236.

Platt, R.R, G.W. Cuperus, M.E. Payton, E.L. Bonjour \& 
Gayana 69(2), 2005

K.N. InKSTON. 1998. Integrated pest management perceptions and practices and insect populations in grocery stores in south-central United States. J. Stored Prod. Res. 34: 1-10.

Pysek, P. 2004. The most complete global overview of invasive species in natural areas. Diversity Dist. 10: $505-508$.

RaJendRan, S. \& K.M. ParveEn. 2005. Insect infestation in stored animal products. J. Stored Prod. Res. 41:1-30.

Singh, G., I.P. Kapoor, S.K. Pander, U.K. Singh \& R.K. Singh. 2002. Studies on essential oils. Part 10; antibacterial activity of volatile oils of some spices. Phytother. Res. 16: 680-682.

Silva, G., A. Lagunes \& J. Rodríguez. 2003. Control de Sitophilus zeamais con polvos vegetales solos y en mezclas con Carbonato de calcio en maíz almacenado. Cien. Inv. Ag. 30: 153-160.

Su, H. C. F. 1986. Laboratory evaluation of the toxicity and repellency of coriander (Coriandrum sativum) seed to four species of stored-product insects. J. Ent. Sci. 21: 169-174.

Tapondjou, L., C. Adler, H. Bouda \& D. A. Fontem. 2002. Efficacy of powder and essential oil from Chenopodium ambrosioides leaves as post- harvest grain protectant against six-stored product beetles. J. Stored Prod. Res. 38:395-402.

TAVAREs, G. M. 2002. Bioatividade da erva de santa maría, Chenopodium ambrosioides L. (Chenopodiaceae) en relação a Sitophilus zeamais Mots., 1855 (Coleoptera: Curculionidae). Dissertaçào (Mestrado). Escola Superior de Agricultura Luiz de Queiroz. Paraçicaba, Sao Paulo, Brasil. 59 p.

Throne, J. 1994. Life history of immature maize weevils (Coleoptera: Curculionidae) on corn stored at constant temperatures and relative humidities in the laboratory. Environ. Entomol. 23: 1459-1471.

Trematerra, P. \& A. Sciaterra. 2004. Spatial distribution of some beetles infesting a feed mill with spatiotemporal dynamics of Oryzaephilus surinamensis, Tribolium castaneum and Tribolium confusum. J. Stored Prod. Res. 40: 363-377.

White, N.D.G. \& J.G. Leesch. 1996. Chemical control. pp. 287-330. In B. Subramanyam \& D.W. Hagstrum (Eds.). Integrated management of insects in stored products. Marcel Dekker, New York, USA, 426p.

Woldemichael, G.M., M.P. Singh, W.M. Maiese \& B.N. Timmermann. 2003. Constituents of antibacterial extract of Caesalpinia paraguarienses Burk. Z. Naturforsch 58: 70-75.

Fecha de recepción: 04/03/05

Fecha de aceptación: 24/06/05 